\title{
Descripción de la flora vascular en el sotobosque de un bosque pantanoso y de una pradera antropogénica húmeda de Juncus procerus en el valle del río Cisnes, Región de Aysén, Chile
}

\section{Description of the vascular flora in the understory of a swamp forest and in an anthropogenic Juncus procerus wet prairie in the valle del río Cisnes, Aysén Region, Chile}

\author{
Alvaro Promis ${ }^{1 *}$, Giselle Bergh ${ }^{1}$, María Teresa Serra ${ }^{1,2} \&$ Gustavo Cruz ${ }^{1}$ \\ ${ }^{1}$ Departamento de Silvicultura y Conservación de la Naturaleza, Universidad de Chile, Casilla 9206, Santiago, Chile. \\ ${ }^{2}$ Consultora Privada. \\ *alvaro.promis@gmail.com
}

\begin{abstract}
The richness, abundance and density of vascular flora species were evaluated in the understory of a swamp forest (BP) dominated by Luma apiculata and in an anthropogenic Juncus procerus wet praire (PHJ). The most diverse taxonomical groups were fern in BP and dycotiledon in PHJ. Introduced species were only found in PHJ. Epiphytic hemicryptophytes and climbing phanerophytes are indicator species for $\mathrm{BP}$ and hemicryptophyes for $\mathrm{PHJ}$.
\end{abstract}

Los bosques pantanosos (BPs) de Chile son humedales dominados principalmente por mirtáceas nativas, ubicándose en depresiones del terreno, con suelos anegados casi todo el año, con bajas tasas de descomposición y mineralización de la hojarasca (Ramírez et al. 1983, San Martín et al. 1988, Ramírez et al. 1996, González et al. 2003). En la zona centro-sur de Chile, estos BPs se encuentran dominados por fanerófitas y hemicriptófitas, siendo las fanerófitas trepadoras un componente importante de su flora vascular (Ramírez et al. 1996). La mayoría de los BPs se encuentran alterados por la acción antrópica (actividad agropecuaria y forestal) (González et al. 2003, Correa-Araneda et al. 2011). La alteración antrópica de estos BPs por sucesivas quemas, roces, pastoreo intenso y drenaje han producido un remplazo en su estructura, transformándolos en matorrales de quila, pantanos de cortadera o praderas de Juncus procerus E. Mey, dificultando su regeneración natural y su potencial restauración (Ramírez et al. 1996, Ramírez et al. 2003).

En la Región de Aysén, desde comienzos del siglo XX se quemaron más de tres millones de hectáreas de bosques para habilitación de praderas agrícolas (Quintanilla 2008). Además, la sobreexplotación y las sucesivas quemas y roces de los BPs han producido el reemplazo de éstos por las ya nombradas praderas de juncáceas, ciperáceas y/o renovales de escaso valor económico, conocidos en la zona como "mallines" (Cruces et al. 1999).

De los estudios realizados en Chile sobre humedales, ninguno se ha enfocado en estudiar los BPs de la Región de
Aysén (Correa-Araneda et al. 2011). Este estudio de caso tiene como objetivo caracterizar la riqueza, diversidad y composición de especies de la flora vascular presentes en un BP (15 ha) y en una pradera antropogénica húmeda de junquillo (PHJ, sensu San Martín et al. 2002) de 13 ha, localizados a unos $20 \mathrm{~km}$ al este de Puerto Cisnes (44 ${ }^{\circ} 42^{\text {' }}$ S; $72^{\circ}$ 33' O; 60 ms.n.m.) (Fig. 1). La PHJ se encuentra contigua al BP, y era parte del BP, bosque que en los años 60 fue cortado y quemado por su propietario para habilitación de terreno para ganadería. En el bosque pantanoso se observaron algunos tocones antiguos de Pilgerodendron uviferum (D.Don.) Florin, lo que evidencia una antigua intervención maderera.

Para evaluar la composición de especies y la estructura del BP se distribuyeron aleatoriamente 5 parcelas circulares de $500 \mathrm{~m}^{2}$. En cada una de las parcelas se identificó la especie y se midió el diámetro a 1,3 m (DAP) a todos los árboles con $\mathrm{DAP} \geq 5 \mathrm{~cm}$. Para evaluar la composición florística, dentro del BP y en la PHJ se distribuyeron aleatoriamente 6 y 10 parcelas de $6 \mathrm{~m}^{2}(3 \times 2 \mathrm{~m})$ respectivamente. El tamaño de la parcela se basó en el método del área mínima (MuellerDombois \& Ellenberg 1974). En cada una de las parcelas se registró la cobertura de todas las plantas vasculares utilizando la escala de Londo (Londo 1984). Solo fueron consideradas las plantas vasculares del sotobosque (plantas que se encontraban creciendo sobre el piso, madera en descomposición, base de árboles y raíces y epífitas sobre los árboles hasta una altura de 1,3 m del suelo). Además 
de los inventarios fitosociológicos se realizaron colectas al azar de aquellas plantas vasculares del sotobosque que no fueron encontradas dentro del muestreo (Hauenstein et al. 2002). La nomenclatura taxonómica sigue lo propuesto por Zuloaga et al. (2009).

El BP tiene una densidad promedio (Dha) de 1.168 árboles/ha y un área basal (Gha) de $26,8 \mathrm{~m}^{2} /$ ha. La distribución de los árboles por clase diamétrica se ajusta a una estructura de bosque multietáneo (Fig. 2). Una alta densidad de individuos de Amomyrtus luma (Molina) Legrand et Kausel se distribuyen en las clases diamétricas inferiores (60,3\% de Dha y 24,6\% de Gha). Nothofagus nitida (Phil.) Krasser domina las clases diamétricas superiores (11,6\% de Dha y 53,9\% de Gha), con árboles emergentes de gran tamaño (DAP sobre $60 \mathrm{~cm}$ y alturas de hasta $18 \mathrm{~m})$.

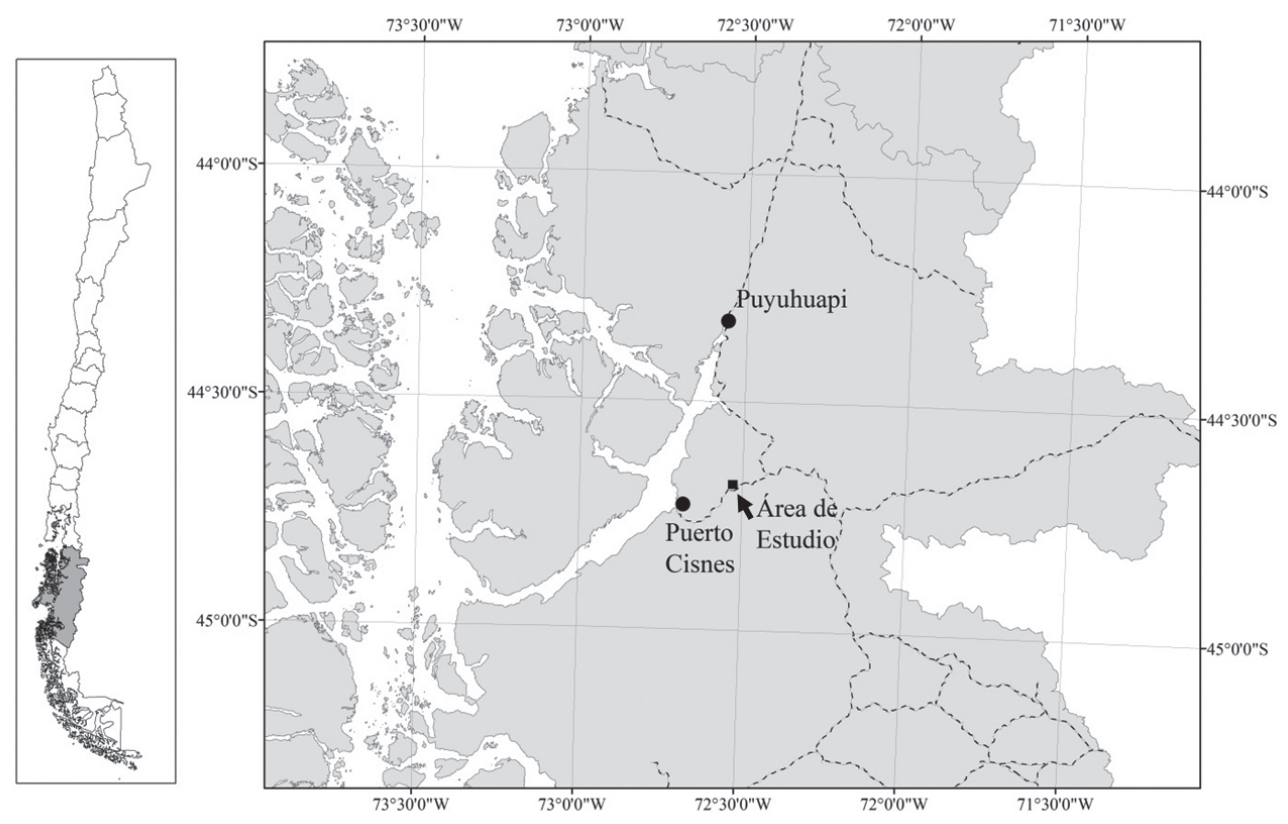

Figura 1. Mapa de ubicación del área de estudio cerca de Puerto Cisnes, Región de Aysén, Chile.

Figure 1. Map showing the study area near to Puerto Cisnes, Aysén Region, Chile.

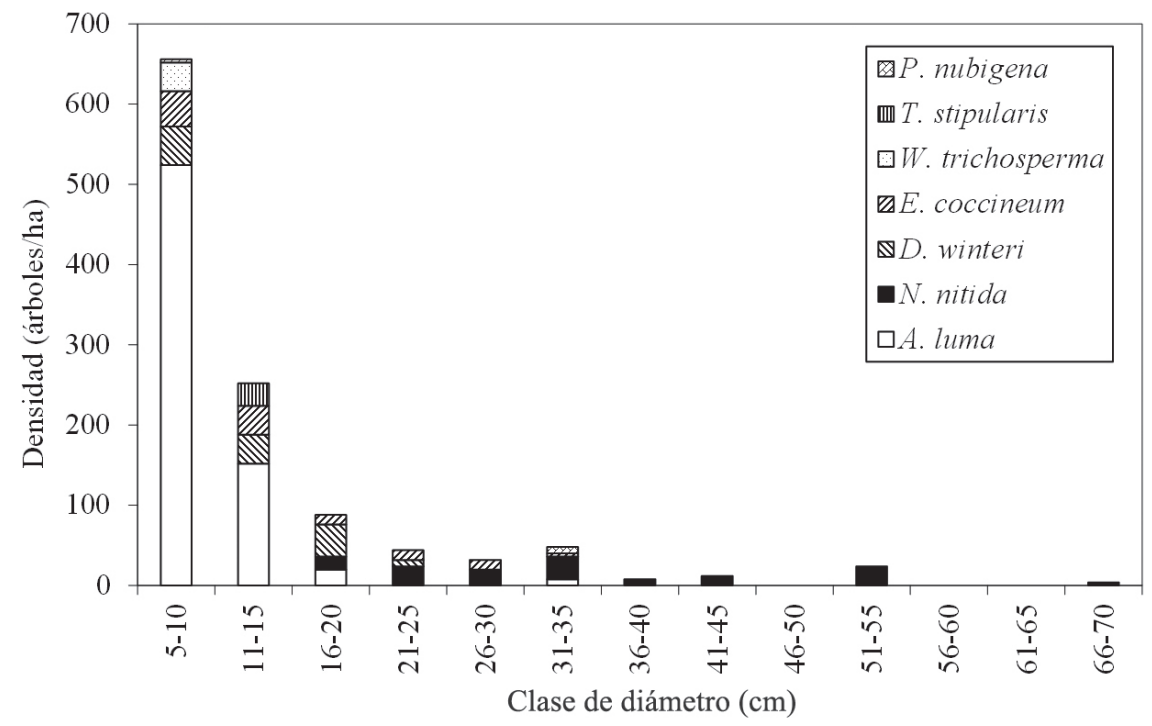

FiguRA 2. Distribución de árboles según clases de diámetro (Clases cada $5 \mathrm{~cm}$, para árboles $\geq 5 \mathrm{~cm}$ de DAP).

Figure 2. Tree diametric distribution per $5 \mathrm{~cm}$ diameter class for trees $\geq 5 \mathrm{~cm} \mathrm{DBH}$. 
En el sotobosque de BP se encontró un total de 42 especies de plantas vasculares, y en PHJ 37 especies (Anexo 1). Ambas formaciones tienen 18 especies comunes (Anexo 1). La mayor riqueza de especies (número de especies promedio por parcela) fue encontrada en BP con 14 especies/parcela y PHJ con 11,6 especies/parcela) (Tabla I). La diversidad de especies también fue mayor en BP (índice de diversidad de Simpson 0,80) que en PHJ (Tabla I). Además, en BP se encontró una mayor abundancia de especies (Tabla I).

En BP el grupo taxonómico mejor representado es el de helechos (53\% de todas las especies presentes), siguen las dicotiledóneas (38\%), las monocotiledóneas (7\%) y las gimnospermas (2\%). En PHJ el grupo taxonómico mejor representado es el de las dicotiledóneas (54\%), luego las monocotiledóneas $(30 \%)$ y por último helechos $(16 \%)$, con ausencia de gimnospermas. En ambas formaciones vegetales se registraron especies propuestas en alguna categoría de conservación (Anexo 1), todas helechos.

Respecto del origen fitogeográfico de las especies (Fig. 3 y Anexo 1), en ambos ambientes la mayoría de las especies son autóctonas. En BP se encontró una especie endémica, Luzuriaga polyphylla (Hook.) J.F. Macbr., y no se registraron especies introducidas (alóctonas). En PHJ se encontraron dos especies alóctonas, Holcus lanatus L. y Carex canescens L. (Anexo 1).

En ambas formaciones vegetales, las formas de vida predominante corresponden a las hemicriptófita y hemicriptófita epífita, con un total de 23 y 17 especies en el BP y en PHJ, respectivamente (Fig. 3 y Anexo 1), siendo una de ellas alóctona en PHJ. Las especies leñosas (fanerófitas, nanofanerófitas y fanerófitas trepadoras) alcanzan a un total de 19 y 17 especies en BP y PHJ, respectivamente. Debe destacarse la gran cantidad de especies hemicriptófita epífitas y trepadoras en el BP (11 especies) con respecto a PHJ (5 especies). Se contabilizaron tres especies terófitas que sólo fueron encontradas en PHJ, siendo una de ellas alóctona (Fig. 3 y Anexo 1).

Las especies asociadas al sotobosque derivada mediante un análisis de indicador de especies, mostró que 13 especies son indicadoras del sotobosque del BP (Tabla II), siendo las formas de vida más representadas las fanerófitas trepadoras con 5 especies y las hemicriptófitas epífitas con 4 especies. De estas especies indicadoras, 5 se encuentran propuestas en alguna categoría de conservación (Anexo 1). En contraste, en PHJ, el número de especies indicadoras disminuyó a 5 , siendo las formas de vida más representadas la hemicriptófita.
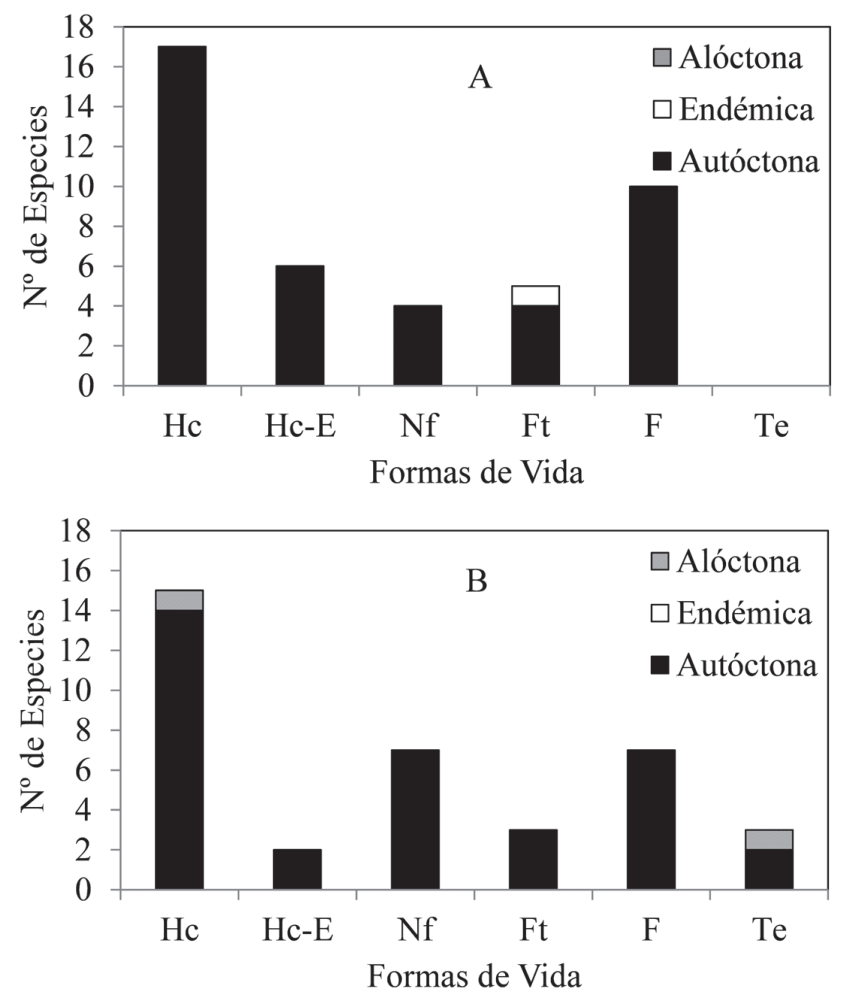

FIgURA 3. Formas de vida y origen fitogeográfico de la flora vascular de A) bosque pantanoso y B) pradera húmeda de junquillo. Hc $=$ Hemicriptófito, $\mathrm{Hc}-\mathrm{E}=$ Hemicriptófita epífita, $\mathrm{Nf}=$ Nanofanerófito, $\mathrm{Ft}=$ Fanerófito trepador, $\mathrm{F}=$ Fanerófito, Te $=$ Terófito.

FIGURE 3. Life form and geographic origin of the vascular flora of the A) swamp forest and B) junquillo's wet prairie. Hc= Hemicryptophyte, $\mathrm{Hc}-\mathrm{E}=$ Hemicryptophyte and epiphyte, $\mathrm{Nf}=$ Nanophanerophyte, $\mathrm{Ft}=$ Climber phanerophytes, $\mathrm{F}=$ Phanerophythe, $\mathrm{Te}=\mathrm{Therophyte}$. 
Flora en bosque pantanoso y pradera húmeda de Juncus procerus: Promis, A. ET AL.

TABLA I. Riqueza, diversidad y abundancia de plantas vasculares en el bosque pantanoso (BP) y en la pradera húmeda de Juncus procerus (PHJ). En paréntesis se encuentran los rangos.

TABLE I. Vascular plant richness, diversity and abundance in the swamp forest (BP) and the Juncus Procerus wet prairie (PHJ). Ranges are in brackets.

\begin{tabular}{|c|c|c|}
\hline & $\mathrm{BP}$ & PHJ \\
\hline \multicolumn{3}{|l|}{ Riqueza de plantas Vasculares } \\
\hline Especies promedio por parcela & $\begin{array}{c}14,0 \\
(10-18)\end{array}$ & $\begin{array}{c}11,6 \\
(8-18)\end{array}$ \\
\hline \multicolumn{3}{|l|}{ Diversidad de plantas Vasculares } \\
\hline Promedio del índice de diversidad de Simpson & $\begin{array}{c}0,80 \\
(0,77-0,82)\end{array}$ & $\begin{array}{c}0,52 \\
(0,26-0,77)\end{array}$ \\
\hline \multicolumn{3}{|l|}{ Abundancia de plantas Vasculares } \\
\hline Promedio de la cobertura de especies por parcela (\%) & 100 & $\begin{array}{c}97 \\
(85-100)\end{array}$ \\
\hline
\end{tabular}

TABLA II. Análisis de indicador de especies para la composición de plantas vasculares del sotobosque del bosque pantanoso y la pradera antropizada húmeda de junquillo. IV es el valor indicador observado obtenido a partir del análisis de indicador de especies. * indica que existe significancia a nivel de un $5 \% \mathrm{y} * *$ a nivel de $1 \%$. FV es la forma de vida según Raunkiaer $(\mathrm{Hc}=\mathrm{Hemicriptófito,} \mathrm{Hc-E}=$ Hemicriptófito epífito, $\mathrm{Nf}=$ Nanofanerófito, $\mathrm{F}=$ Fanerófito, $\mathrm{Ft}=$ Fanerófito trepador).

TABLE II. Indicator species analysis for the understorey vascular species composition in the swamp forest and the human disturbed junquillo's wet prairie. IV is the observed indicator value obtained from the indicator species analysis. * indicates significance at the $5 \%$ level, and $* *$ at the $1 \%$ level. FV is the Raunkiaer plant life form $(\mathrm{Hc}=$ Hemicryptophyte, $\mathrm{Hc}-\mathrm{E}=$ Epiphytic hemicryptophyte, $\mathrm{Nf}=\mathrm{Nanophanerophyte,}$ $\mathrm{F}=$ Phanerophyte, $\mathrm{Ft}=$ Climbing phanerophyte).

\begin{tabular}{|c|c|c|c|c|c|c|c|}
\hline \multicolumn{4}{|c|}{ Bosque Pantanoso } & \multicolumn{4}{|c|}{ Pradera HÚMEDA DE JunQUILlo } \\
\hline ESPECIES & IV & $\mathrm{p}$ & $\mathrm{FV}$ & ESPECIES & IV & $\mathrm{p}$ & $\mathrm{FV}$ \\
\hline Grammitis magellanica & 50,0 & $0,041^{*}$ & $\mathrm{Hc}$ & Carex darwinii & 70,0 & $0,034^{*}$ & $\mathrm{Hc}$ \\
\hline Hymenophyllum ferrugineum & 50,0 & $0,029 *$ & Hc-E & Gunnera magellanica & 80,0 & $0,006 * *$ & $\mathrm{Hc}$ \\
\hline Hymenophyllum pectinatum & 76,3 & $0,006^{* *}$ & Hc-E & Juncus procerus & 70,0 & $0,019^{*}$ & $\mathrm{Hc}$ \\
\hline Hymenophyllum tortuosum & 65,4 & $0,018^{*}$ & Hc-E & Nertera granadensis & 60,0 & $0,035^{*}$ & $\mathrm{Hc}$ \\
\hline Serpyllopsis caespitosa & 50,0 & $0,041^{*}$ & Hc-E & Nothofagus nitida & 60,0 & $0,045^{*}$ & $\mathrm{~F}$ \\
\hline Chusquea spp. & 81,0 & $0,001 * *$ & $\mathrm{Nf}$ & & & & \\
\hline Asteranthera ovata & 66,7 & $0,009 *$ & $\mathrm{Ft}$ & & & & \\
\hline Campsidium valdivianum & 71,4 & $0,017^{*}$ & $\mathrm{Ft}$ & & & & \\
\hline Luzuriaga polyphylla & 66,7 & $0,018^{*}$ & $\mathrm{Ft}$ & & & & \\
\hline Mitraria coccinea & 72,2 & $0,029 *$ & $\mathrm{Ft}$ & & & & \\
\hline Philesia magellanica & 61,4 & $0,035^{*}$ & $\mathrm{Ft}$ & & & & \\
\hline Amomyrtus luma & 82,4 & $0,009 * *$ & $\mathrm{~F}$ & & & & \\
\hline Drimys winteri & 99,7 & $0,001 * *$ & $\mathrm{~F}$ & & & & \\
\hline
\end{tabular}




\section{AGRADECIMIENTOS}

Se agradece al proyecto FPA "Conservación de mallines. Una alternativa para proteger humedales, educación ambiental y desarrollo de actividades ecoturísticas". Al apoyo en la descripción de especies por los ingenieros forestales Patricio Medina y Hernán Retamales

\section{BIBLIOGRAFÍA}

Baeza, M., E. Barrera, J. Flores, C. Ramírez \& R. Rodríguez. 1998. Categorías de Conservación de Pteridophyta nativas de Chile. Boletín del Museo Nacional de Historia Natural (Chile) 47: 23 - 46.

Correa-Araneda, F., J. Urrutia \& R. Figueroa. 2011. Estado del conocimiento y principales amenazas de los humedales boscosos de agua dulce en Chile. Revista Chilena de Historia Natural 84: 325-340.

Cruces, P., J. Cerda \& M. Ahumada. 1999. Guías de condición para los pastizales de la ecorregión templada húmeda de Aysén. SAG, Coyhaique, Chile. 137 pp.

González, M., E. Hauenstein, F. Peña-Cortés, M. García \& O. URRUTIA. 2003. Comentarios sobre bosques pantanosos, humedales importantes del Centro-Sur de Chile. Gestión Ambiental 9: 3-13.

Hauenstein, E., M. González, F. Peña-Cortés, F. \& A. MuñozPedreros. 2002. Clasificación y caracterización de la flora y vegetación de los humedales de la costa de Toltén (IX Región, Chile). Gayana Botánica 59(2): 87-100.

Londo, G. 1984. The decimal scale for releves of permanent quadrats. En: R. Knapp (ed.), Sampling methods and taxon analysis in vegetation science. pp. 45-49. Dr. W. Junk Publishers, The Hague, The Netherlands.

Mueller-Dombois, D. \& H. Ellenberg. 1974. Aims and Methods of Vegetation Ecology. John Wiley \& Sons, Inc., New York, USA. 547 pp.

Quintanilla, V. 2008. Perturbaciones a la vegetación nativa por grandes fuegos de 50 años atrás, en bosques nordpatagónicos. Caso de estudio en Chile Meridional. Anales de Geografía 28(1): 85-104.

Ramírez, C., F. Ferreire \& H. Figueroa. 1983. Estudio fitosociológico de los bosques pantanosos templados del sur de Chile. Revista Chilena de Historia Natural 56: 11-26.

Ramírez, C., C. San Martín \& J. SAn Martín. 1996. Estructura florística de los bosques pantanosos de Chile SurCentral. En: J.J. Armesto, C. Villagrán \& M.K. Arroyo (eds.), pp. 215-234, Editorial Universitaria, Santiago, Chile.

Ramírez, C., J. Amigo \& C. San Martín. 2003. Vegetación pratense litoral y dinámica vegetacional antropogénica en Valdivia, Chile. Agro Sur 31: 24-37.

San Martín, C., C. Ramírez \& H. Rubilar. 2002. Ecosociología de los pantanos de cortadera en Valdivia, Chile. Ciencia e Investigación Agraria 29(3): 171-179.

San Martín, J., A. Troncoso \& C. Ramírez. 1988. Estudio fitosociológico de los bosques pantanosos nativos de la Cordillera de la Costa en Chile central. Bosque 9(1): 1733.

Zuloaga, F., O. Morrone \& M. Belgrano. 2009. Catálogo de las Plantas Vasculares del Cono Sur. Versión base de datos en sitio web del Instituto Darwinion, Argentina. URL: http://www2.darwin.edu.ar/Proyectos/FloraArgentina/ FA.asp

AnEXo 1. Catálogo de la flora vascular del bosque pantanoso (BP) y pradera húmeda de junquillo (PHJ).

ANNEX 1. List of the vascular plant species of the swamp forest (BP) and junquillo's wet prairie (PHJ)

\begin{tabular}{|c|c|c|c|c|c|}
\hline Clase/Nombre Científico & FAMILIA & $\mathrm{FV}$ & Of & Fm & Ec \\
\hline \multicolumn{6}{|l|}{ FILICOPSIDA } \\
\hline Asplenium dareoides Desv. & Aspleniaceae & $\mathrm{Hc}$ & A & BP-PHJ & $\mathrm{FP}(1)$ \\
\hline Blechnum cordatum (Desv.) Hieron. & Blechnaceae & $\mathrm{Hc}$ & $\mathrm{A}$ & $\mathrm{BP}^{*}$ & $\mathrm{FP}(1)$ \\
\hline Blechnum magellanicum (Desv.) Mett & Blechnaceae & $\mathrm{Hc}$ & A & BP- PHJ & \\
\hline Blechnum penna-marina (Poir.) Kuhn & Blechnaceae & $\mathrm{Hc}$ & A & BP- PHJ & \\
\hline Cystopteris fragilis (L.) Bernh. & Dryopteridaceae & $\mathrm{Hc}$ & A & $\mathrm{PHJ} *$ & \\
\hline Grammitis magellanica Desv. & Grammitidaceae & $\mathrm{Hc}$ & A & $\mathrm{BP}$ & $\mathrm{V}(1)$ \\
\hline Grammitis poeppigiana (Mett.) Pic. Ser. & Grammitidaceae & $\mathrm{Hc}$ & A & $\mathrm{BP} *$ & $\mathrm{IC}(1)$ \\
\hline Hymenoglossum cruentum (Cav.) K. Presl & Hymenophyllaceae & $\mathrm{Hc}$ & A & $\mathrm{BP}^{*}$ & $\mathrm{~V}(1)$ \\
\hline Hymenophyllum cuneatum Kunze & Hymenophyllaceae & $\mathrm{Hc}$ & A & $\mathrm{BP}^{*}$ & $\mathrm{~V}(1)$ \\
\hline Hymenophyllum dentatum Cav. & Hymenophyllaceae & $\mathrm{Hc}$ & A & $\mathrm{BP}^{*}$ & $\mathrm{FP}(1)$ \\
\hline Hymenophyllum ferrugineum Colla & Hymenophyllaceae & Hc-E & A & $\mathrm{BP}$ & $\mathrm{FP}(1)$ \\
\hline Hymenophyllum krauseanum Phil. & Hymenophyllaceae & Hc-E & A & $\mathrm{BP} *$ & \\
\hline Hymenophyllum pectinatum Cav. & Hymenophyllaceae & Hc-E & A & BP- PHJ & $\mathrm{FP}(1)$ \\
\hline Hymenophyllum plicatum Kaulf. & Hymenophyllaceae & $\mathrm{Hc}$ & A & $\mathrm{BP}^{*}$ & \\
\hline Hymenophyllum secundum Hook. et Grev. & Hymenophyllaceae & Hc-E & A & $\mathrm{BP}^{*}$ & \\
\hline Hymenophyllum seselifolium K. Presl & Hymenophyllaceae & $\mathrm{Hc}$ & A & $\mathrm{BP}^{*}$ & \\
\hline Hymenophyllum tortuosum Hook. et Grev. & Hymenophyllaceae & Hc-E & A & BP- PHJ & $\mathrm{V}(1)$ \\
\hline Lophosoria quadripinnata (J.F. Gmel.) C. Chr. & Dicksoniaceae & $\mathrm{Hc}$ & A & $\mathrm{BP} *$ & $\mathrm{~V}(1)$ \\
\hline Polystichum plicatum (Poepp. ex Kunze) Hicken. & Dryopteridaceae & $\mathrm{Hc}$ & A & $\mathrm{BP}$ & \\
\hline
\end{tabular}


Flora en bosque pantanoso y pradera húmeda de Juncus procerus: Promis, A. ET AL.

Serpyllopsis caespitosa (Gaud.) C. Chr. var. caespitosa

Sticherus cfr. cryptocarpus (Hook.) Ching

Sticherus quadripartitus (Poir.) Ching

Synammia feuillei (Bertero) Copel. var. feuillei

PINOPSIDA

Podocarpus nubigena Lindl.

\section{MAGNOLIOPSIDA}

Amomyrtus luma (Molina) Legrand et Kausel

Asteranthera ovata (Cav.) Hanst.

Berberis darwinii Hook.

Berberis microphylla G. Forst.

Campsidium valdivianum (Phil.) Skottsb.

Drimys winteri J.R. Forst. et G. Forst.

Embothrium coccineum J.R. Forst. et G. Forst.

Escallonia rosea Griseb.

Fuchsia magellanica Lam.

Gaultheria mucronata (L.f.) Gaud. ex Spreng.

Griselinia ruscifolia (Clos) Ball

Gunnera magellanica Lam.

Gunnera tinctoria (Molina) Mirb.

Laureliopsis philippiana (Looser) Schodde

Leptinella scariosa Cass.

Lomatia ferruginea (Cav.) R. Br.

Mitraria coccinea Cav.

Nertera granadensis (Mutis ex L.f.) Druce

Nothofagus betuloides (Mirb.) Oerst.

Nothofagus nitida (Phil.) Krasser

Ranunculus minutiflorus Bert. ex Phil.

Raukaua laetevirens (Gay) Frodin

Rubus geoides J.E. Sm.

Tepualia stipularis (Hook. et Arn.) Griseb.

Weinmannia trichosperma Cav.

\section{LILIOPSIDA}

Carex acutata Boott

Carex canescens $\mathrm{L}$.

Carex chillanensis Phil.

Carex darwinii Boott

Chusquea spp.

Holcus lanatus L.

Isolepis cernua (Vahl.) Roem. et Schult.

Juncus bufonius L.

Juncus procerus E. Mey.

Luzuriaga polyphylla (Hook.) J.F. Macbr.

Philesia magellanica J.F. Gmel.

Polypogon australis Brongn.

$\begin{array}{lllll}\text { Hymenophyllaceae } & \text { Hc-E } & \text { A } & \text { BP } & \text { IC(1) } \\ \text { Gleicheniaceae } & \text { Hc } & \text { A } & \text { BP* } & \\ \text { Gleicheniaceae } & \text { Hc } & \text { A } & \text { BP* } & \text { FP(1) } \\ \text { Polypodiaceae } & \text { Hc } & \text { A } & \text { BP* } & \\ & & & & \\ \text { Podocarpaceae } & \text { F } & \text { A } & \text { BP } & \end{array}$

Myrtaceae

Gesneriaceae

Berberidaceae

Berberidaceae

Bignoniaceae

Winteraceae

Proteaceae

Escalloniaceae

Onagraceae

Ericaceae

Griseliniaceae

Gunneraceae

Gunneraceae

Monimiaceae

Asteraceae

Proteaceae

Gesneriaceae

Rubiaceae

Nothofagaceae

Nothofagaceae

Ranunculaceae

Araliaceae

Rosaceae

Myrtaceae

Cunoniaceae

$\mathrm{F}$
$\mathrm{Ft}$
$\mathrm{Nf}$
$\mathrm{Nf}$
$\mathrm{Ft}$
$\mathrm{F}$
$\mathrm{F}$
$\mathrm{Nf}$
$\mathrm{Nf}$
$\mathrm{Nf}$
$\mathrm{Nf}$
$\mathrm{Hc}$
$\mathrm{Hc}$
$\mathrm{F}$
$\mathrm{Hc}$
$\mathrm{F}$
$\mathrm{Ft}$
$\mathrm{Hc}$
$\mathrm{F}$

$\begin{array}{llll}\text { Cyperaceae } & \mathrm{Hc} & \text { A } & \text { PHJ } \\ \text { Cyperaceae } & \mathrm{Hc} & \text { AL } & \text { PHJ } \\ \text { Cyperaceae } & \mathrm{Hc} & \text { A } & \text { PHJ * } \\ \text { Cyperaceae } & \mathrm{Hc} & \text { A } & \text { PHJ } \\ \text { Poaceae } & \mathrm{Nf} & \text { A } & \text { BP- PHJ } \\ \text { Poaceae } & \text { Te } & \text { AL } & \text { PHJ * } \\ \text { Cyperaceae } & \text { Te } & \text { A } & \text { PHJ * } \\ \text { Juncaceae } & \text { Te } & \text { A } & \text { PHJ } \\ \text { Juncaceae } & \mathrm{Hc} & \text { A } & \text { PHJ } \\ \text { Luzuriagaceae } & \mathrm{Ft} & \text { E } & \text { BP } \\ \text { Philesiaceae } & \mathrm{Ft} & \text { A } & \text { BP- PHJ } \\ \text { Poaceae } & \mathrm{Hc} & \text { A } & \text { PHJ }\end{array}$

$\mathrm{FV}=$ forma de vida según Raunkiaer $(\mathrm{Hc}=$ Hemicriptófito, $\mathrm{Hc}-\mathrm{E}=$ Hemicriptófito epífito, $\mathrm{Nf}=$ Nanofanerófito, $\mathrm{F}=\mathrm{Fanerófito}, \mathrm{Ft}=$ Fanerófito trepador)

Of $=$ origen fitogeográfico $(\mathrm{A}=$ Autóctono, $\mathrm{AL}=$ Alóctono y $\mathrm{E}:$ Endémico $)$

$\mathrm{Fm}=$ Formación vegetal $(\mathrm{BP}=$ bosque pantanoso, $\mathrm{PHJ}=$ pradera húmeda de junquillo)

$\mathrm{Ec}=$ Estado de Conservación $(\mathrm{V}=$ Vulnerable, $\mathrm{IC}=$ Insuficientemente Conocida, FP = Fuera de Peligro $)$

* Especie encontrada fuera de muestreo

(1) Baeza et al. (1998).

Recibido: 12.04 .12

Aceptado: 30.01 .13 